\title{
Relation between preoperative autonomic function and blood pressure change after tourniquet deflation during total knee replacement arthroplasty
}

In Young Huh, Dae-Young Kim, Ji-Hyeon Lee, Soo Jin Shin, Young Woo Cho, and Soon Eun Park

Department of Anesthesiology and Pain Medicine, Ulsan University Hospital, Ulsan, Korea

Background: Tourniquets are used to provide a bloodless surgical field for extremities. Hypotension due to vasodilation and bleeding after tourniquet deflation is a common event. Hemodynamic stability is modulated by the autonomic nervous system (ANS). Heart rate variability (HRV) is a sensitive method for detecting individuals who may be at risk of hemodynamic instability during general anesthesia. The purpose of this study was to investigate ANS function to predict hypotension after tourniquet deflation.

Methods: Eighty-six patients who underwent total knee replacement arthroplasty (TKRA) were studied. HRV, systolic blood pressure variability (SBPV) and baroreflex sensitivity (BRS) were analyzed. We assigned two groups depending on the lowest systolic blood pressure (SBP) or mean BP (MBP) after tourniquet release (Group H; SBP $<80 \mathrm{mmHg}$ or MBP $<60 \mathrm{mmHg}$, Group S; SBP $>80 \mathrm{mmHg}$ and MBP $>60 \mathrm{mmHg}$ ).

Results: Fifteen patients developed severe hypotension and ten patients were treated with ephedrine. Of the

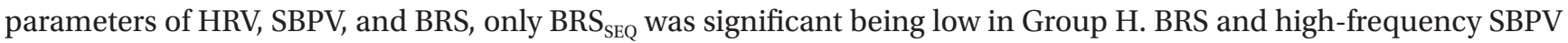
were correlated with the degree of MBP change after tourniquet deflation.

Conclusions: Preoperative low BRS is associated with hypotension after tourniquet deflation, suggesting the importance of baroreflex regulation for intraoperative hemodynamic stability. (Korean J Anesthesiol 2012; 62: 154-160)

Key Words: Baroreflex sensitivity, Heart rate variability, Hypotension, Systolic blood pressure variability, Tourniquet deflation.

Received: October 25, 2011. Revised: 1st, November 10, 2011; 2nd, November 12, 2011. Accepted: November 14, 2011.

Corresponding author: In Young Huh, M.D., Department of Anesthesiology and Pain Medicine, Ulsan University Hospital, 290-3, Jeonha-dong Dong-gu, Ulsan 682-714, Korea. Tel: 82-52-250-7248, Fax: 82-52-250-7249, E-mail: inyoung_huh@uuh.ulsan.kr

(c) This is an open-access article distributed under the terms of the Creative Commons Attribution Non-Commercial License (http:// creativecommons.org/licenses/by-nc/3.0/), which permits unrestricted non-commercial use, distribution, and reproduction in any medium, provided the original work is properly cited. 


\section{Introduction}

The pneumatic tourniquet is an integral part of the surgical procedure of the extremities [1]. Deflation of the tourniquet causes hypotension and the release of metabolites from the ischemic limb, and cardiac arrest may occur after tourniquet deflation in an unusual event [2,3]. No study has been attempted to evaluate the degree of decrease of blood pressure and the predictive factor of hypotension, despite the incidence of hypotension after tourniquet deflation. Intraoperative hemodynamic instability is a major concern for anesthesiologists during general anesthesia. Especially, it has been suggested that in elderly patients the mortality risk increases when the duration of hypotension becomes sufficiently long [4]. Therefore, determining the independent risk factor of hypotension may be of paramount importance for anesthetists when preparing elderly patients for anesthesia.

Systemic hemodynamic regulation is controlled by the autonomic nervous system (ANS) [5,6]. Noninvasive methods of measuring the activity of ANS include the analysis of heart rate variability (HRV), systolic blood pressure variability (SBPV) and baroreflex sensitivity (BRS) [6-8]. Studies have shown the predictive value of HRV for hypotension in patients undergoing caesarean section with spinal anesthesia or after induction of general anesthesia $[9,10]$. Hence, preoperative determination of autonomic regulation may be provided to detect a patient's risk of significant hemodynamic instability.

This study was designed to evaluate the predictive power of HRV, SBPV and BRS for tourniquet hypotension in patients undergoing total knee replacement arthroplasty (TKRA). We hypothesized that preoperative HRV, SBPV, and BRS differ between patients depending on the severity of hypotension after tourniquet deflation during TKRA.

\section{Materials and Methods}

After approval from the Institutional Ethics Committee of our Hospital, and written informed consents were obtained, eighty six patients scheduled to undergo TKRA during general anesthesia were studied. Not all patients received oral premedication but continued their medication for their underlying disease.

Patients were allowed to acclimatize for at least $10 \mathrm{~min}$ in the supine position in a quiet operating room at an ambient temperature. The radial artery was cannulated with a 22-guaze catheter, and after 10 minutes, the baseline beat-to-beat electrocardiography (ECG) and arterial blood pressure (BP) signal were recorded for 5 minutes before administration of any sedatives. Induction and maintenance of anesthesia were performed in a standardized manner in all patients. Anesthesia was induced with etomidate $0.2 \mathrm{mg} / \mathrm{kg}$ as a bolus and maintained with sevoflurane inhalation. Tracheal intubation was facilitated by infusion of rocuronium bromide $0.8 \mathrm{mg} / \mathrm{kg}$. Remifentanil was continuously infused at $0.15 \mu \mathrm{g} / \mathrm{kg} / \mathrm{min}$ until the trachea was intubated and then at $0.05-0.15 \mu \mathrm{g} / \mathrm{kg} / \mathrm{min}$ to maintain blood pressure at $\pm 20 \%$ of preoperative value. Depth of anesthesia was monitored using the Bispectral Index (BIS) (BIS XP, Aspect Medical systems, Norwood, USA) aiming at a BIS level of under 60 . If the BIS value exceeded 60 , sevoflurane was increased stepwise by $0.2 \mathrm{vol} \%$.

HRV, SBPV and BRS analysis were performed according to a previous report [11]. Beat-by-Beat ECG and arterial BP signals (Intellivue MP70, Phillps Medizinsystems, Boebligen, Germany) were digitized and collected at 500 samples per second using an online personal computer that interfaced with an analog-todigital converter (DI-720U, DARAQ instruments, Akron, OH). Offline analysis was performed using signal processing software (CODAS, DATAQ;DADiSP/Adv DSP, DSP Development, Cambridge, MA) and custom-written MATLAB (The MathWoks, Inc., Natick, MA) scripts.

The R-R interval (RRI) was derived from the time difference between marks placed on the peaks of the $\mathrm{R}$ waves and systolic BP (SBP) was derived from the maxima of beat-by-beat arterial waveforms. Time-domain analysis of HRV was calculated from the root mean squared successive difference of RRI (RMSSD) and the percentage of successive adjacent RRI greater than 50 ms (pNN50). These parameters are the most frequently used and are thought to be related to the parasympathetic control of the heart [7]. For frequency domain analysis, 5 minutes data of beat-by-beat RRI and SBP were interpolated to $5 \mathrm{~Hz}$ to provide equidistant samples. The fast Fourier transformed power spectrum density, based on Welch's algorithm of averaging periodograms, was calculated for the filtered signals using a sliding window with a width of 500 points and an overlap of 250 points after detrending and application of Hanning window. The resulting five periodograms were averaged to produce the estimated spectrum. This method yields a frequency resolution of $0.01 \mathrm{~Hz}$. The areas under the power spectrum in the low frequency (LF) and high frequency (HF) regions (defined as $0.04-0.15 \mathrm{~Hz}$ and $0.15-0.4 \mathrm{~Hz}$, respectively) of HRV and SBPV were integrated [7]. In addition, the LF and HF components of HRV were measured in normalized units, which represent the relative value of each power component in proportion to the total power of the LF and HF bands. The HF power of HRV was used as a valid marker of vagal tone, and LF was considered as a sympathetic and parasympathetic control of heart rate [7]. SBPV was performed in the same manner as that of the frequency domain of HRV. The LF power of SBPV was used as an index of vascular sympathetic outflow and HF power was used as an index of the mechanical effect of respiration $[6,12]$. 
BRS was estimated from spontaneous beat-by-beat fluctuations in SBP and RRI. This study engaged frequency domain transfer function analysis and the time domain sequence method for quantifying BRS $[11,13,14]$. Briefly, the transfer function magnitude and coherence (the squared coherence function) between SBP and RRI were estimated using the cross spectral method [13]. We calculated the magnitude between HRV and SBPV separately as an index of BRS in the $\mathrm{LF}\left(\mathrm{BRS}_{\mathrm{LF}}\right)$ and $\mathrm{HF}\left(\mathrm{BRS}_{\mathrm{HF}}\right)$ regions, where coherence is more than 0.5. The sequence analysis identifies sequences of 3 or more consecutive heartbeats, in which both SBP and RRI concordantly increase or decrease, with the threshold at $1 \mathrm{mmHg}$ and $4 \mathrm{~ms}$, respectively [14]. The linear regression was applied between SBP and RRI. The regression slope was calculated for those sequences with the squared correlation coefficients exceeding 0.85 . The sequence BRS $\left(\mathrm{BRS}_{\mathrm{SEQ}}\right)$ reflects the average regression slope for all the linear regressions plotted for the accepted baroreflex sequence within the 5 minutes data. The pneumatic tourniquet was inflated to $300 \mathrm{mmHg}$ around the thigh prior to incision and was released after the TKRA instrument insertion. Patients were assigned to one of two groups depending on BP change followed by defining hypotension. BP was recorded as an absolute value. After tourniquet deflation, hypotension was defined as absolute MBP $<60 \mathrm{mmHg}$ or the lowest SBP $<80 \mathrm{mmHg}$ (H group, S group; $\mathrm{SBP} \geq 80 \mathrm{mmHg}$ and $\mathrm{MBP} \geq 60 \mathrm{mmHg}$ ). Hypotension

Table 1. Patients Medical Characteristics and Preoperative Medications

\begin{tabular}{lccc}
\hline & Group S & Group H & P value \\
\hline Group number & 71 & 15 & \\
Sex $(\mathrm{M} / \mathrm{F})$ & $8 / 63$ & $1 / 14$ & \\
Age $(\mathrm{yr})$ & $67.7 \pm 5.2$ & $68.1 \pm 4.5$ & 0.471 \\
Height $(\mathrm{cm})$ & $152.8 \pm 7.5$ & $151.3 \pm 6.0$ & 0.612 \\
Weight $(\mathrm{kg})$ & $62.0 \pm 10.9$ & $63.2 \pm 8.1$ & 0.466 \\
Body mass index $\left(\mathrm{kg} / \mathrm{m}^{2}\right)$ & $26.5 \pm 4.0$ & $27.5 \pm 2.2$ & 0.213 \\
Ejection fraction $(\%)$ & $64.4 \pm 9.4$ & $58.9 \pm 18.7$ & 0.149 \\
Underlying disease & & & \\
Hypertension & $33(46.5 \%)$ & $7(46.7 \%)$ & 0.992 \\
Diabetes mellitus & $14(19.7 \%)$ & $4(26.7 \%)$ & 0.593 \\
Coronary artery disease & $4(5.6 \%)$ & $0(0 \%)$ & 0.358 \\
Cerebrovascular accidents & $2(2.8 \%)$ & $0(0 \%)$ & 0.516 \\
Medication & & & \\
Oral hypoglycemic agent & $10(14.1 \%)$ & $3(20 \%)$ & 0.592 \\
Insulin & $1(1.4 \%)$ & $1(6.7 \%)$ & 0.225 \\
Calcium channel blocker & $30(42.3 \%)$ & $5(33.3 \%)$ & 0.623 \\
Beta blocker & $17(23.9 \%)$ & $4(26.7 \%)$ & 0.846 \\
ACEI & $7(9.9 \%)$ & $3(20 \%)$ & 0.295 \\
Aspirin & $22(31.0 \%)$ & $5(33.3 \%)$ & 0.883 \\
Diuretics & $4(5.6 \%)$ & $1(6.7 \%)$ & 0.880 \\
Isorbide dinitrate & $4(5.6 \%)$ & $0(0 \%)$ & 0.358 \\
\hline
\end{tabular}

Values are mean \pm SD and numbers of patients (\%). Comparisons between the two groups were conducted using student t-test, Fisher's exact test, or Chi-square test. Group S: $\mathrm{SBP} \geq 80 \mathrm{mmHg}$, and $\mathrm{MBP} \geq$ $60 \mathrm{mmHg}$, Group H: SBP $<80 \mathrm{mmHg}$, or MBP $<60 \mathrm{mmHg}$, ACEI: Angiotensin converting enzyme inhibitors. was treated in a standardized manner with $5 \mathrm{mg}$ ephedrine and fluid replacement, repeated if necessary. The drug was administrated until MBP increased to $60 \mathrm{mmHg}$ or greater. The amount of medication was recorded. HRV, SBPV and BRS were analyzed depending on the patient's group.

Comparisons between groups were made using students t-test for normally distributed continuous variables and the Mann-Whitney U-test for continuous variables that were not normally distributed. Pearson's correlation analysis was used to examine the correlations between hemodynamic variables and the degree of BP change after tourniquet deflation. Chi-square or the Fisher' exact test were used to compare the incidence of hypotension in patients with underlying disease or medication. To examine the predictors of the incidence of hypotension, logistic regression using the stepwise forward elimination procedure was performed. A P value $<0.05$ was considered a significant difference.

\section{Results}

Fifteen patients of the study participants experienced hypotension after tourniquet release, and ten patients were treated with ephedrine $(7 \pm 3.50 \mathrm{mg})$. Patient medical characteristics and preoperative medications are shown in Table 1. Intraoperative anesthetic characteristics are listed in Table 2.

A t-test and Mann-Whitney U-test analysis of all the variables showed that the two groups significantly differed in BRS ${ }_{\mathrm{SEQ}}$,

Table 2. Intraoperative Anesthetic Management

\begin{tabular}{lccc}
\hline & Group S & Group H & P value \\
\hline Tourniquet time (min) & $83.7 \pm 15.1$ & $86.3 \pm 13.6$ & 0.589 \\
Fluid replacement before & & & \\
release & & & \\
$\quad$ Crystalloid (ml) & $1,490.2 \pm 475.0$ & $1,609.1 \pm 200.9$ & 0.408 \\
$\quad$ Colloid (ml) & $307.1 \pm 150.4$ & $240.0 \pm 177.2$ & 0.308 \\
Mean blood pressure & & & \\
(mmHg) & & & \\
Before release & $96.4 \pm 10.5$ & $94.5 \pm 11.0$ & 0.724 \\
After release & $74.9 \pm 8.5$ & $57.5 \pm 3.9 *$ & $<0.001$ \\
MBP change (\%) & $21.9 \pm 7.5$ & $38.5 \pm 6.7 *$ & $<0.001$ \\
Sevoflurane (vol\%) & & & \\
Before release & $1.79 \pm 0.33$ & $1.91 \pm 0.27$ & 0.141 \\
After release & $1.08 \pm 0.25$ & $0.97 \pm 0.10^{*}$ & 0.012 \\
Remifentanil ( $\mu$ g/kg/min) & & & \\
Before release & $0.08 \pm 0.04$ & $0.09 \pm 0.03$ & 0.438 \\
After release & $0.03 \pm 0.02$ & $0.01 \pm 0.01 *$ & 0.037 \\
BIS (bispectral index) & & & \\
Before release & $39.8 \pm 5.5$ & $40.8 \pm 5.4$ & 0.701 \\
After release & $42.3 \pm 5.9$ & $43.7 \pm 4.4$ & 0.782 \\
\hline
\end{tabular}

Values are mean \pm SD. Comparisons between the two groups were conducted using the student t-test. Group S: SBP $\geq 80 \mathrm{mmHg}$, and $\mathrm{MBP} \geq 60 \mathrm{mmHg}$, Group H: SBP $<80 \mathrm{mmHg}$, or MBP $<60 \mathrm{mmHg}$, MBP: mean blood pressure. ${ }^{*} \mathrm{P}<0.05$ is the statistically significant difference between the two groups. 
Table 3. Perioperative Cardiovascular Variables

\begin{tabular}{|c|c|c|c|}
\hline & Group S & Group H & $P$ value \\
\hline RRI (ms) & $866.1 \pm 172.7$ & $846.0 \pm 167.5$ & 0.681 \\
\hline \multicolumn{4}{|l|}{ Heart rate variability } \\
\hline $\operatorname{VLF}\left(\mathrm{Ln} \mathrm{ms} \mathrm{s}^{2}\right)$ & $5.50 \pm 1.15$ & $5.41 \pm 1.10$ & 0.769 \\
\hline $\left.\mathrm{LF}(\mathrm{Ln} \mathrm{ms})^{2}\right)$ & $4.72 \pm 1.21$ & $4.54 \pm 1.35$ & 0.524 \\
\hline $\mathrm{HF}\left(\mathrm{Ln} \mathrm{ms}^{2}\right)$ & $3.76 \pm 1.44$ & $3.68 \pm 1.38$ & 0.861 \\
\hline nuLF & $0.68 \pm 0.20$ & $0.65 \pm 0.26$ & 0.932 \\
\hline nuHF & $0.32 \pm 0.20$ & $0.35 \pm 0.26$ & 0.932 \\
\hline LF/HF ratio & $4.02 \pm 3.78$ & $4.51 \pm 4.81$ & 0.932 \\
\hline RMSSD (Ln ms) & $2.68 \pm 0.77$ & $2.60 \pm 0.65$ & 0.735 \\
\hline pNN50 (Ln, \%) & $0.55 \pm 1.26$ & $0.63 \pm 1.99$ & 0.814 \\
\hline \multicolumn{4}{|c|}{ Systolic blood pressure variability } \\
\hline SBP (mmHg) & $163.8 \pm 17.4$ & $165.9 \pm 23.7$ & 0.909 \\
\hline $\mathrm{LF}\left(\mathrm{Ln} \mathrm{mmHg}^{2}\right)$ & $2.65 \pm 0.62$ & $2.76 \pm 0.71$ & 0.546 \\
\hline $\mathrm{HF}\left(\mathrm{Ln} \mathrm{mmHg}^{2}\right)$ & $0.57 \pm 0.85$ & $0.97 \pm 0.98$ & 0.123 \\
\hline \multicolumn{4}{|c|}{ Baroreflex sensitivity (BRS) } \\
\hline $\mathrm{BRS}_{\mathrm{LF}}(\mathrm{ms} / \mathrm{mmHg})$ & $5.50 \pm 3.74$ & $4.12 \pm 2.62$ & 0.249 \\
\hline $\mathrm{BRS}_{\mathrm{HF}}(\mathrm{ms} / \mathrm{mmHg})$ & $6.44 \pm 6.21$ & $4.29 \pm 3.05$ & 0.344 \\
\hline $\mathrm{BRS}_{\mathrm{SEQ}}(\mathrm{ms} / \mathrm{mmHg})$ & $5.29 \pm 3.81$ & $3.11 \pm 2.32^{*}$ & 0.044 \\
\hline
\end{tabular}

Values are mean \pm SD. Group S: SBP $\geq 80 \mathrm{mmHg}$, and MBP $\geq 60 \mathrm{mmHg}$, Group H: SBP < $80 \mathrm{mmHg}$, or MBP < $60 \mathrm{mmHg}$, RRI: R-R intervals, VLF: very low frequency power, LF: low-frequency power, HF: high-frequency power, Ln: natural logarithmic-transformed power, nuLF: normalized low-frequency unit, nuHF: normalized high-frequency unit, $\mathrm{BRS}_{\mathrm{LF}}$ : baroreflex sensitivity at low-frequency, $\mathrm{BRS}_{\mathrm{HF}}$ : baroreflex sensitivity at highfrequency, $\mathrm{BRS}_{\mathrm{SEQ}}$ : baroreflex sensitivity by sequence method. Comparisons between the two groups were conducted using student $\mathrm{t}$-test. ${ }^{*} \mathrm{P}<0.05$ is the statistically significant difference between the two groups.
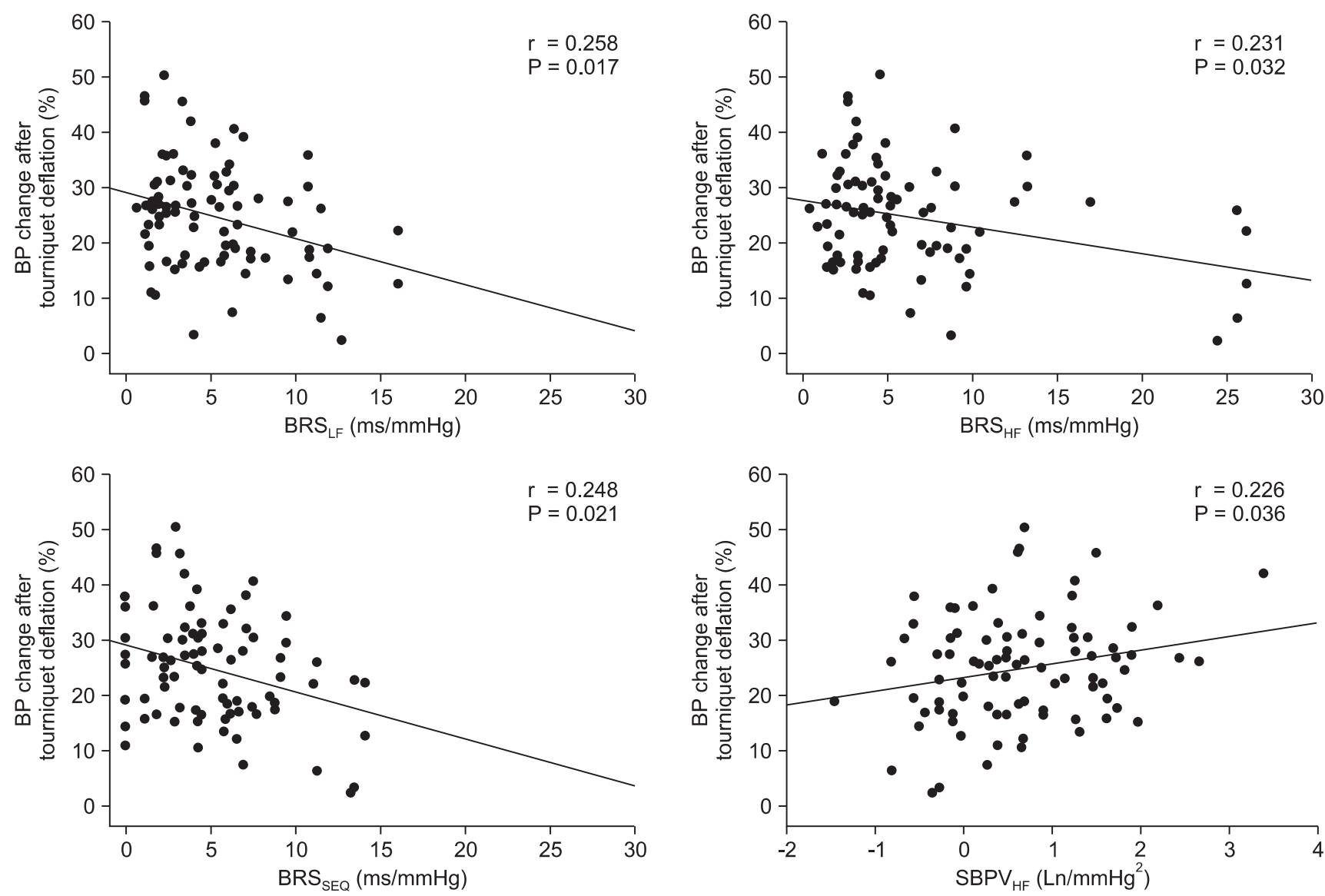

Fig. 1. Correlation between $\mathrm{BP}$ change after tourniquet deflation and BRS and $\mathrm{SBPV}_{\mathrm{HF}}$. BRS is negatively correlated and $\mathrm{SBPV}_{\mathrm{HF}}$ is positively correlated with blood pressure change after tourniquet release in total knee replacement arthroplasty. BRS: baroreflex sensitivity, LF: lowfrequency, HF: high-frequency, SEQ: sequence method, $\mathrm{SBPV}_{\mathrm{HF}}$ : $\mathrm{HF}$ band in systolic blood pressure variability. 
whereas other variables were similar (Table 3). Correlation analysis revealed that the extent of MBP change after tourniquet deflation negatively correlated with $\mathrm{BRS}_{\mathrm{LF}}(\mathrm{r}=0.258, \mathrm{p}=0.017)$, $\mathrm{BRS}_{\mathrm{HF}}(\mathrm{r}=0.231, \mathrm{p}=0.032)$, and $\mathrm{BRS}_{\mathrm{SEQ}}(\mathrm{r}=0.248, \mathrm{p}=0.021)$, and positively correlated with $\mathrm{SBPV}_{\mathrm{HF}}(\mathrm{r}=0.226, \mathrm{p}=0.036)$ (Fig. 1). However, the frequency domain variables of HRV and SBPV, except $\mathrm{SBP}_{\mathrm{HF}}$ were not related with the extent of MBP reduction after tourniquet release.

The univariate linear regression and multiple logistic regression analysis were not performed because the incidence rate was not sufficient and the power of the performed test is below the desired power (0.8).

\section{Discussion}

In this study, we investigated the relation between preoperative autonomic function and blood pressure change after pneumatic tourniquet deflation during TKRA. Fifteen patients (17\%) were assigned to the $\mathrm{H}$ group after tourniquet deflation. The degree of BP change after tourniquet deflation is related to $\mathrm{SBPV}_{\mathrm{HF}}$ and BRS. The severe hypotension after tourniquet release is not predicted by preoperative autonomic function as measured by HRV, SBPV and BRS.

Hypotension is a well-known complication of tourniquet deflation and is associated with decrease systemic vascular resistance due to the removal of occlusion of artery, bleeding because of unligated vessel, release of ischemic tissue metabolite and hypovolemia [2]. The recovery of hypotension after tourniquet deflation is faster in general anesthesia than regional anesthesia, because of the blockade of the sympathetic nervous system $[2,15]$. There is evidence that hemodynamic instability during general anesthesia is associated with adverse outcomes in patients having non-cardiac disease $[4,16]$. Monk et al. [16] proved three independent factors for 1-year mortality following surgical procedure, thereafter one of the predictors was intra-operative hypotension. In that study, they found a relative mortality risk increase with $3.6 \%$ for every minute of hypotension that was defined by an SBP of less than $80 \mathrm{mmHg}$ [16].

Systemic hemodynamic regulation is controlled by the ANS. Analysis of HRV, SBPV, and BRS is a noninvasive, indirect measure of autonomic regulation [7,12-14]. Alternation of ANS has also been reported in various medical conditions, such as aged person, hypertension, myocardial infarction and diabetes $[6,17]$. Latson et al. [17] documented that some degree of ANS dysfunction was common in patients older than 39 years old, and such dysfunction is related to an increased incidence of hypotension after anesthesia induction. We considered that some degree of ANS dysfunction was frequently developed in our study population. However, we did not compare this with younger people and we did not perform provocative tests, such as Valsalva maneuver and deep breathing test [7].

HRV is based on measuring the beat-to-beat interval of the sinus rhythm and might offer more insight into the physiological mechanisms of autonomic cardiovascular control $[7,8,18]$. RMSSD, pNN50, and HF reflect the parasympathetic activity of heart rate modulation and the LF is influenced by the baroreceptor system that reflects the sympathetic as well as parasympathetic nervous system and the LF/HF ratio reflects a parameter of sympatho-vagal balance $[7,18]$. Recently, HRV has been shown to be a reliable tool to predict hypotension after the induction of general anesthesia or spinal anesthesia $[9,10,19]$. In this study, HRV did not differ between the two groups, and there was also no correlation between HRV and BP change after tourniquet deflation. This study reported conflicting results with the other study. We assumed that underlying medical conditions and medications may have affected HRV. Diabetes and medications, such as beta-blocker, calcium channel blocker, or angiotensin converting enzyme inhibitor, include exclusion criteria in other studies $[9,10]$. There was no difference between the two groups in underlying disease and medications in the present study, and these factors were neglected in both groups. However, the relationship between the duration of underlying disease and HRV cannot be discussed, because this information was not available in the present study. Our study population was older than that of other studies, and therefore the decreased HRV might not provide discrimination. Other studies are concerned with the relation of HRV and hemodynamic change at induction or after spinal anesthesia, which are considered more vital change than tourniquet deflation $[9,10,19]$.

SBPV is equally important to HRV, but remains largely unknown in general anesthesia. SBPV elicited by sympathetic modulation of vascular tone occurs in the LF band and respiration induced mechanical effect produces the $\mathrm{HF}$ band [20]. SBPV $\mathrm{HF}_{\mathrm{HF}}$ was positively correlated with MBP change after tourniquet release in the present study. $\mathrm{SBPV}_{\mathrm{HF}}$ reflects the stroke volume change by changing intrathoracic pressure during respiration and is closely related to HF in HRV [20]. Although it is not well defined in spontaneous respiration, systolic pressure variation (SPV), the difference between maximal and minimal systolic pressure during single mechanical breath, has been known to be a cardiac preload, and it is considered as $\mathrm{SBPV}_{\mathrm{HF}}$ $[20,21]$. Therefore, greater $\mathrm{SBPV}_{\mathrm{HF}}$ may reflect a reduction of preload in the preoperative period and consequently induce a greater BP change after tourniquet deflation. However, we should evaluate the effectiveness of $\mathrm{SBPV}_{\mathrm{HF}}$ and SPV as preload indexes in spontaneous breathing.

BRS is a powerful negative feedback reflex that attempts to control the fluctuation of $\mathrm{BP}$. The primary mechanism of BP homeostasis by BRS is through the effects on both the para- 
sympathetic and sympathetic branches of ANS [22]. Baroreflex abnormalities have been described early in the course of diabetes and hypertension [22,23]. Several methods are available to assess the BRS, and we engaged two distinct methods for assessing BRS, the sequence method and transfer function $[13,14,24]$. $\mathrm{BRS}_{\mathrm{LF}}, \mathrm{BRS}_{\mathrm{HF}}$, and $\mathrm{BRS}_{\mathrm{SEQ}}$ were negatively correlated with MBP change after tourniquet deflation and BRS $_{\mathrm{SEQ}}$ was significantly lower in patients who developed hypotension than in those who did not. It has been suggested that lower BRS induces greater BP change, because BRS relates to homeostasis in BP. Namely, a greater BRS means a greater ability of maintenance BP and may induce less change of BP after tourniquet deflation, though under the sevoflurane anesthesia. Sevoflurane significantly depressed BRS [25], and therefore we should assess the BRS during anesthesia and before tourniquet deflation.

There were several limitations to this study. First, preoperative cardiovascular variables are obtained in the operation room before surgery. Thus, withholding sedatives may be allowed to contribute to hypertension and tachycardia and to induce the alteration of these parameters. Second, 89.5 percent of participants were old-aged females, and therefore the present results may not extrapolate to other populations. Third, our study includes a small number of patients with underlying diseases. These diseases may be a confounding factor of the result, even though incidence of disease did not differ in this study. Further study should consider the diabetes and myocardial infarction, etc. Finally, we did not examine the relation between postoperative complication and intraoperative hypotension. Hypotension after tourniquet deflation is transient, but the extent of hypotension may be affected by postoperative delirium or cardiac event in an old aged person.

In summary, our data supports the premise that BRS and $\mathrm{SBPV}_{\mathrm{HF}}$ are correlated with blood pressure change after tourniquet release. Preoperative low BRS is associated with hypotension after tourniquet deflation, suggesting the importance of baroreflex regulation for intraoperative hemodynamic stability. Future studies will be required to ascertain how to predict hypotension of tourniquet deflation in preoperative hemodynamic variables, such as HRV, SBPV, and BRS.

\section{Acknowledgements}

This work was funded by Ulsan University Hospital (Biomedical Reserach Center Promotion Fund).

\section{References}

1. Gielen MJ, Stienstra R. Tourniquet hypertension and its prevention: a review. Reg Anesth 1991; 16: 191-4.
2. Townsend HS, Goodman SB, Schurman DJ, Hackel A, Brock-Utne JG. Tourniquet release: systemic and metabolic effects. Acta Anaesthesiol Scand 1996; 40: 1234-7.

3. McGrath BJ, Hsia J, Epstein B. Massive pulmonary embolism following tourniquet deflation. Anesthesiology 1991; 74: 618-20.

4. Bijker JB, van Klei WA, Vergouwe Y, Eleveld DJ, van Wolfswinkel L, Moons KG, et al. Intraoperative hypotension and 1-year mortality after noncardiac surgery. Anesthesiology 2009; 111: 1217-26.

5. Akselrod S, Gordon D, Ubel FA, Shannon DC, Berger AC, Cohen RJ. Power spectrum analysis of heart rate fluctuation: a quantitative probe of beat-to-beat cardiovascular control. Science 1981; 213: 220-2.

6. Pagani M, Lucini D. Autonomic dysregulation in essential hypertension: insight from heart rate and arterial pressure variability. Auton Neurosci 2001; 90: 76-82.

7. Heart rate variability: standards of measurement, physiological interpretation and clinical use. Task Force of the European Society of Cardiology and the North American Society of Pacing and Electrophysiology. Circulation 1996; 93: 1043-65.

8. Bootsma M, Swenne CA, Van Bolhuis HH, Chang PC, Cats VM, Bruschke AV. Heart rate and heart rate variability as indexes of sympathovagal balance. Am J Physiol 1994; 266: H1565-71.

9. Hanss R, Bein B, Ledowski T, Lehmkuhl M, Ohnesorge H, Scherkl W, et al. Heart rate variability predicts severe hypotension after spinal anesthesia for elective cesarean delivery. Anesthesiology 2005; 102 : 1086-93.

10. Huang CJ, Kuok CH, Kuo TB, Hsu YW, Tsai PS. Pre-operative measurement of heart rate variability predicts hypotension during general anesthesia. Acta Anaesthesiol Scand 2006; 50: 542-8.

11. Kim YK, Hwang GS, Huh IY, Hwang JH, Park JY, Chung SL, et al. Altered autonomic cardiovascular regulation after combined deep and superficial cervical plexus blockade for carotid endarterectomy. Anesth Analg 2006; 103: 533-9.

12. Parati G, Saul JP, Di Rienzo M, Mancia G. Spectral analysis of blood pressure and heart rate variability in evaluating cardiovascular regulation. A critical appraisal. Hypertension 1995; 25: 1276-86.

13. Saul JP, Berger RD, Albrecht P, Stein SP, Chen MH, Cohen RJ. Transfer function analysis of the circulation: unique insights into cardiovascular regulation. Am J Physiol 1991; 261: H1231-45.

14. Rothlisberger BW, Badra LJ, Hoag JB, Cooke WH, Kuusela TA, Tahvanainen KU, et al. Spontaneous 'baroreflex sequences' occur as deterministic functions of breathing phase. Clin Physiol Funct Imaging 2003; 23: 307-13.

15. Modig J, Kolstad K, Wigren A. Systemic reactions to tourniquet ischaemia. Acta Anaesthesiol Scand 1978; 22: 609-14.

16. Monk TG, Saini V, Weldon BC, Sigl JC. Anesthetic management and one-year mortality after noncardiac surgery. Anesth Analg 2005; 100: 4-10.

17. Latson TW, Ashmore TH, Reinhart DJ, Klein KW, Giesecke AH. Autonomic reflex dysfunction in patients presenting for elective surgery is associated with hypotension after anesthesia induction. Anesthesiology 1994; 80: 326-37.

18. Malliani A, Pagani M, Lombardi F, Cerutti S. Cardiovascular neural regulation explored in the frequency domain. Circulation 1991; 84: 482-92.

19. Hanss R, Renner J, Ilies C, Moikow L, Buell O, Steinfath M, et al. 
Does heart rate variability predict hypotension and bradycardia after induction of general anaesthesia in high risk cardiovascular patients? Anaesthesia 2008; 63: 129-35.

20. Toska K, Eriksen M. Respiration-synchronous fluctuations in stroke volume, heart rate and arterial pressure in humans. J Physiol 1993; 472: 501-12.

21. Perel A, Pizov R, Cotev S. Systolic blood pressure variation is a sensitive indicator of hypovolemia in ventilated dogs subjected to graded hemorrhage. Anesthesiology 1987; 67: 498-502.

22. Lanfranchi PA, Somers VK. Arterial baroreflex function and cardio- vascular variability: interactions and implications. Am J Physiol Regul Integr Comp Physiol 2002; 283: R815-26.

23. Ewing DJ, Clarke BF. Diagnosis and management of diabetic autonomic neuropathy. Br Med J (Clin Res Ed) 1982; 285: 916-8.

24. Parati G, Frattola A, Di Rienzo M, Castiglioni P, Pedotti A, Mancia G. Effects of aging on 24-h dynamic baroreceptor control of heart rate in ambulant subjects. Am J Physiol 1995; 268: H1606-12.

25. Umehara S, Tanaka M, Nishikawa T. Effects of sevoflurane anesthesia on carotid-cardiac baroreflex responses in humans. Anesth Analg 2006; 102: 38-44. 